(C) 2017 IEEE. Personal use of this material is permitted. Permission from IEEE must be obtained for all other uses, in any current or future media, including reprinting/republishing this material for advertising or promotional purposes, creating new collective works, for resale or redistribution to servers or lists, or reuse of any copyrighted component of this work in other works. 


\title{
Lightweight Authentication Protocol (LAUP) for 6LoWPAN Wireless Sensor Networks
}

\author{
Annie Gilda Roselin ${ }^{1,2}$ \\ Priyadarsi Nanda ${ }^{1}$ \\ Surya Nepal
Surya.Nepal@data61.csiro.au \\ Annie.G.ArockiaBaskaran@student.uts.edu.au \\ Priyadarsi.Nanda@uts.edu.au
}

1. School of Computing and Communications, Faculty of Engineering and IT, University of Technology Sydney, Australia. 2. CSIRO/Data61, Marsfield, Sydney, Australia.

\begin{abstract}
LoWPAN networks involving wireless sensors consist of resource starving miniature sensor nodes. Since secured authentication of these resource-constrained sensors is one of the important considerations during communication, use of asymmetric key distribution scheme may not be the perfect choice to achieve secure authentication. Recent research shows that Lucky Thirteen attack has compromised Datagram Transport Layer Security (DTLS) with Cipher Block Chaining (CBC) mode for key establishment. Even though EAKES6Lo and S3K techniques for key establishment follow the symmetric key establishment method, they strongly rely on a remote server and trust anchor for secure key distribution. Our proposed Lightweight Authentication Protocol (LAUP) used a symmetric key method with no preshared keys and comprised of four flights to establish authentication and session key distribution between sensors and Edge Router in a 6LWPAN environment. Each flight uses freshly derived keys from existing information such as PAN ID (Personal Area Network IDentification) and device identities. We formally verified our scheme using the Scyther security protocol verification tool for authentication properties such as Aliveness, Secrecy, Non-Injective Agreement and Non-Injective Synchronization. We simulated and evaluated the proposed LAUP protocol using COOJA simulator with ContikiOS and achieved less computational time and low power consumption compared to existing authentication protocols such as the EAKES6Lo and SAKES.
\end{abstract}

\section{INTRODUCTION}

The network of low power sensors called LoWPAN (Low Power Wireless Personal Area Network) consists of small sensors with limited memory, less computational capability and low in resources. 6LoWPAN is one of the most important communication protocols used in LoWPAN network. 6LoWPAN is designed in such a way that it can transmit the IPv6 packets over LoWPAN network [1] [2] [3] [4]. Security in LoWPAN is provided through authentication of sensor device before any communication happens.

Last MAC and Handshake Authentication methods are mainly used to provide anti-replay protection and authentication [5] for low power devices. The MAC value (hashed value of the message and key) of the previous message is appended to the current message, and the receiver validates the received MAC with the already stored MAC value. However, this technique assumes that the receiver will always accept the first message packet and globally shared keys for authentication. Even though ECC based Diffie-Hellman key exchange with Kerberos authentication [6] for wireless sensor networks provide high security, symmetric encryption and handshake authentication are the highly desirable mechanisms to build the authentication for the successive message transmissions of LoWPAN devices. The public key method of key distribution requires more computational time and energy which leads to overhead for lightweight sensors.

The plaintext of LoWPAN communication can be recovered from a DTLS connection using an OpenSSL implementation of DTLS when using CBC (Cipher Block Chaining) mode encryption [7]. So it is not a good idea to have DTLS for Key management otherwise DTLS-CBC mode has to be enhanced to manage Lucky thirteen type attacks. To overcome these attacks, LAUP uses ECB (Electronic Code Book) mode of AES128 encryption. Moreover, compressed DTLS has six flights for authentication, whereas our proposed LAUP has only four flights for authentication and session key distribution.

Existing mechanisms such as lightweight IKEv2, EAKES6Lo, S3K and compressed DTLS are providing lightweight authentication for wireless sensor communication. Lightweight IKEv2 [8] is secure but requires more memory for calculation. EAKES6Lo [9] uses hash functions to ensure the integrity of messages. Though this technique uses symmetric key cryptography, it assumes that the secret key distributed to every node by the remote server. The distribution of secret keys remains a challenge.

S3K symmetric key establishment for IoT [8] uses trust anchor and a resource server for key distribution among clients. However, the secret key is shared between trust anchor and resource server before deployment. The limitation of this idea is that it is not scalable for a vast network and mobility nodes. Maintaining the uniqueness of shared key between trust anchor and resource server is complicated. S3K runs in addition to DTLS and CoAP protocols which in turn, increases the computational overhead of LoWPAN devices. Moreover, the secure connection has to be established between trust anchor and remote server before the key generation process using TLS/DTLS.

After a thorough study on key distribution and authentication of 6LoWPAN networks, we come to the following conclusions. The existing algorithms, which follow pre-shared methods are facing problems while updating the keys and having the assumptions of pre-shared keys. Moreover, they are relying on a key distribution center to distribute the keys 
TABLE I: Theoretical comparison and Features of LAUP

\begin{tabular}{|r|l|l|l|l|l|}
\hline No & Parameters & Compressed DTLS & EAKES6Lo & S3K & LAUP \\
\hline 1 & Asymmetric method & yes & No & No & No \\
\hline 2 & Symmetric + preshared keys & No & Yes & Yes & No \\
\hline 3 & Use of Key Distribution centre & No & Yes & Yes & No \\
\hline 4 & Symmetric + No preshared keys & No & No & No & Yes \\
\hline
\end{tabular}

which in turn cost more resources and providing protection to key distribution center are another problem in real time. Well established existing algorithms which are using asymmetric method for authentication and key distribution are having the limitation of spending more computational time for authentication. To overcome these existing constraints, we proposed our LAUP algorithm for authentication and key establishment. LAUP leads to the highly secured and easily adaptable authentication method for 6LoWPAN networks. Table 1 shows the explanation of our approach to 6LoWPAN networking.

Our proposed LAUP authentication algorithm addresses the significant challenges such as a distribution of preshared keys and usage of resource centers for key distribution in the field of authentication among low power devices. We made observations on how the conventional network protocols compressed to be compatible with LoWPAN Wireless Sensor Networks (WSN). Our observation showed that Compressed DTLS Handshake [10] uses six flights for authentication and key exchange, whereas our protocol uses only four flights for the same. To our knowledge, LAUP authentication algorithm is the most suitable for 6LoWPAN network and secure authentication algorithm without using preshared keys for authentication and key distribution of LoWPAN devices.

The rest of the paper is organized as follows. Section II explains related works in the area of authentication and key distribution of LoWPAN networks. Section III describes our proposed work using session request phase, authentication phase, and key distribution phase. Formal verification using Scyther tool is presented in section IV. Section V analyses the efficiency of the LAUP protocol against various attacks of LoWPAN network. Performance evaluation of LAUP using Contiki OS COOJA simulator is demonstrated in section VI. Finally, we conclude the paper in section VII.

\section{RELATED WORK}

APKES(Adaptive Pairwise Key Establishment Scheme) [11] uses pre-distributed pairwise keys to derive pairwise session keys for authentication. SPINS (Security Protocol for Sensor Networks) [12] scheme provides security for wireless sensor communication also obtains keys from the pre-distributed master keys. Although AKES (Adaptive Key Establishment Scheme) [13] system uses PAN ID and address of the sensor for authentication and key distribution, it follows predistribution of keys to derive pairwise session keys. Unlike our LAUP, AKES uses the address of sensors to get the shared secret keys, whereas LAUP uses MAC ID of sensors.
Moreover, sensors (LoWPAN devices) which are using AKES scheme for authentication, to be preloaded with any of the relevant addressing information like 8-byte extended, 2-byte short or 1-byte simple address. Since LAUP uses MAC ID of devices, it does not need any reloading of address. APKES, SPINS and AKES methods discussed previously, but in most cases, attention directed towards pre-distributed keys for key distribution and authentication.

SAKES [14] and EAKES6Lo authentication schemes deal with pre-shared keys among the 6LoWPAN host, 6LoWPAN router, and 6LoWPAN edge router. EAKES6Lo [9] has three phases such as pre-deployment phase, authentication and key establishment phase and handover phase. The remote server distributes private/public keys used by the sensors during authentication. A registration request by the sensor node is sent to the server with sensor ID and its public key. This public key is derived using ECDH (Elliptic Curve DiffieHelman) mechanism which is more power consuming process for LoWPAN devices. But LAUP eliminates this additional power requirement by not using a remote server for preshared keys. LAUP focuses on preventing attacks on transportation layer such as replay attack, a man in the middle attack, and impersonation attack.

GDP (Group Device Pairing) does not need extra hardware devices for preshared keys. Based on symmetric key cryptographic techniques GDP provides secure communication between wireless body area networks. Even though GDP method [15] supports no redistribution of keys, GDP needs human user intervention for verification during authentication. Periodical updates of local keys [16] could prevent sensor compromisation on static nodes. Smaller cryptographic keys play a significant role in providing security for sensor communication [17]. Unlike GDP, LAUP does not need human intervention during authentication and key establishment process. Moreover, LAUP session keys are small and have a periodical update for each session.

\section{PROPOSED WORK}

Our proposed LAUP provides security to the 6LoWPAN device communication by authenticating the intended 6LoWPAN devices with Edge Router. 6LoWPAN is designed in such a way that it can transmit the IPv6 packets over Low Power Personal Area Network.

6LoWPAN protocol stack adopts bottom-most two layers from IEEE 802.15.4. 6LoWPAN acts as an adaptation layer between the link layer and the network layer. Figure 1 shows 


\begin{tabular}{|c|c|}
\hline APPLICATION & CoAP \\
\hline TRANSPORT & UDP \\
\hline NETWORK & IPv6/RPL \\
\hline ADAPTATION & 6LoWPAN Adaptation \\
\hline MAC & IEEE 802.15.4 \\
\hline PHYSICAL & IEEE 802.15.4 \\
\hline
\end{tabular}

Fig. 1: 6LoWPAN protocol stack

6LoWPAN protocol stack and examples of protocols used in each layer. IEEE 802.15.4 supports only 127-byte packet length of messages. But the Maximum Transferrable Unit (MTU) of IPV6 is 1280 bytes. 6LoWPAN adaptation layer provides fragmentation and re-ordering, and compression of the protocol stack headers of IPv6 packets to maintain the communication compatibility between IEEE 802.15.4 frame and the legacy Internet message packet [1], [18], [19]. LAUP works on transport layer of the 6LoWPAN protocol.

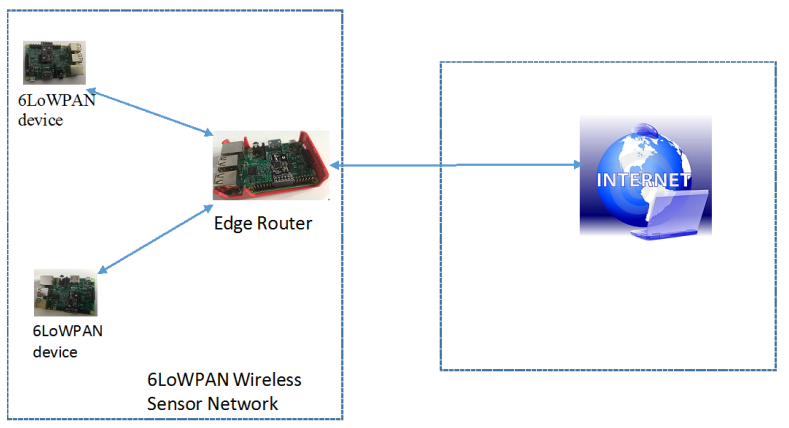

Fig. 2: System architecture

\section{A. Architectural Environment}

System architecture shown in Figure 2 includes 6LoWPAN devices which are intended to communicate with the 6LoWPAN Edge Router. LAUP works on the 6LoWPAN wireless sensor network communication between the 6LoWPAN device and the Edge Router. To maintain the secure communication 6LoWPAN device has to reside on the coverage range of Border Router.

\section{B. Basic assumptions of proposed work}

Every sensor identity $I D_{S}$ (MAC Address of sensor) is registered with the 6LoWPAN Edge Router $\left(6 L_{E R}\right)$ and they are physically secured. Our LAUP deals with 6LoWPAN devices which are deployed within the coverage range of 6LoWPAN Edge Router $6 L_{E R}$. $6 L_{E R}$ knows the PAN ID of LoWPAN network, and we assume that the sensors connected to the LoWPAN network are physically secured. We address
TABLE II: Key Derivation Process

\begin{tabular}{lcr}
\hline Flight No & Key & Process \\
\hline 1 & SK1 & PANID \\
2 & SK2 & $I D_{S}$ XOR Nonce $_{S}$ XOR SK1 \\
3 & SK3 & $I D_{E R}$ XOR SK2 \\
4 & SK4 & Nonce $_{S^{\prime}}$ XOR Nonce XOR SK3 $_{S}$ SK1 XOR SK2 XOR \\
SESSIONKEY & $K_{\text {Session }}$ & SK3 XOR SK4 XOR Nonce RR $^{\prime}$ \\
\hline
\end{tabular}

the authentication and session key establishment of sensors when they are communicating to $6 L_{E R}$.

Each flight calculates its key for session key distribution by following common key derivation method. LAUP does not use any software or hardware based random number generation scheme to produce nonce values. Instead, the time of message generation on each flight has been taken as nonce respectively. By this way of receiving a nonce value, reduces the extra computational complexity and memory usage of low power devices. Table II explains the key derivation method to calculate unique flight keys. These methods use simple XOR functions with available values such as PAN ID, MAC ID and nonce values.

\section{Proposed LAUP algorithm}

LAUP allows sensors of LoWPAN networks to communicate with a router to get cryptographically secure session key by two level authentication using MAC ID of sensors and their nonce values. LAUP algorithm gives protection against a

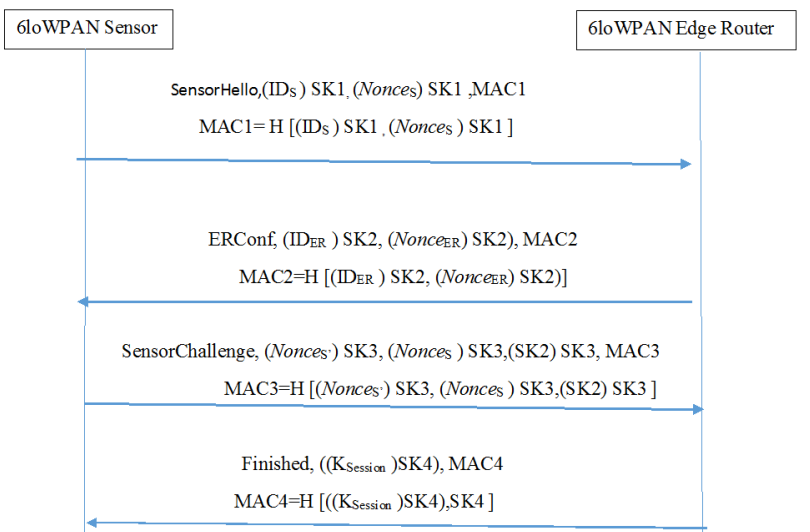

Fig. 3: Proposed Authentication Algorithm

Replay Attack, Man in the Middle attack, and impersonation attack by including the MAC values and nonce values. Even the attacker eavesdropped the message; he can not be able to reproduce the same message since the message is in the encrypted form and it needs the exact time of when the packet was generated. 
As a result of our LAUP algorithm, a unique session key will be produced by the Edge Router for a sensor claims "SensorHello" request. Figure 3 shows the flow of communication of LAUP between 6LoWPAN device and Edge Router. To encrypt the messages in each flight, we use the AES-128-ECB algorithm. A simple XOR function is used as a hash function to produce MAC values in all the four flights of communication. Figure 4 explains the process flow of LAUP on Edge router. Our proposed LAUP algorithm has three phases for authentication and session key establishment process.

1. Session Request

2. Authentication

3. Key Distribution

1) Session Request phase: $6 L_{S} \longrightarrow 6 \mathrm{~L}_{E R}$ :

SensorHello, $\left(I D_{S}\right)$ SK1, $\left(\right.$ Nonce $\left._{S}\right)$ SK1, MAC1

$\mathrm{MACl}=\mathrm{H} \quad\left[\left(I D_{S}\right) \mathrm{SK} 1,\left(\right.\right.$ Nonce $\left.\left._{S}\right) \mathrm{SK} 1\right]$

Session request phase comprises of flight $_{\text {one }}$ communication message. In this phase, the 6LoWPAN sensor which is intended to communicate with the 6LoWPAN Edge Router $6 L_{E R}$ sends the following content in its payload to the $6 L_{E R}$. PAN ID of the network acts as a flight $t_{\text {one }}$ key to encrypt the messages involved in first flight communication. The identity(MAC_ID) and the timer value (time generated by the sensor) of the sensor is encrypted by the flight $_{\text {one }}$ key called SK1. $M A C_{\text {one }}$ value is calculated by applying the XOR function on the encrypted messages. Encrypted identity, encrypted nonce of the sensor, $M A C_{\text {one }}$ value and "SensorHello" message are sent as a first flight information to $6 L_{E R}$. Hence the identity and the nonce value of the sensor is retrieved by the $6 L_{E R}$.

2) Authentication phase: $6 L_{E R} \longrightarrow 6 \mathrm{~L}_{S}$ :

ERConf, $\left(I D_{E R}\right)$ SK2, (Nonce $E R$ ) SK2), MAC2

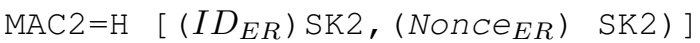

$6 L_{S} \longrightarrow 6 \mathrm{~L}_{E R}:$

Sensorchallenge,

$\left(\right.$ Nonce $\left._{S^{\prime}}\right)$ SK3, (Nonce $\left._{S}\right)$ SK3, (SK2) SK3, MAC3

$\mathrm{MAC} 3=\mathrm{H} \quad\left[\left(\right.\right.$ Nonce $\left._{S^{\prime}}\right) \mathrm{SK} 3,\left(\right.$ Nonce $\left._{S}\right)$

SK3, (SK2) SK3 ]

After receiving the flight $_{\text {one }}$ information from sensor, $M A C_{\text {one }}$ value is calculated at Edge Router by hashing the received encrypted values. Before validating the authenticity of the sensor, the received $M A C_{\text {one }}$ value is compared with the calculated $M A C_{\text {one }}$ value. If both the values are same, protection against replay attack can be ensured, and the authentication process is going to be carried out by the Edge Router.

Two levels of authentication (Initial level and second level) will be performed by the $6 L_{E R}$. Flow chart of Edge Router process in Figure 4 explains the two level authentication process in detail. In the Initial level of authentication, the received flight one information are decrypted using an AES128 algorithm with ECB mode. Retrieved sensor MAC_ID from flight $_{\text {one }}$ information is checked against the already registered sensor MAC_ID. If a match is found, then $6 L_{E R}$ generates flight $_{\text {two }}$ key SK2 by applying the XOR function on sensor MAC_ID, sensor nonce value and flight one $_{\text {key. }}$ Otherwise, 6LER send "You are not a registered sensor" message to the corresponding sensor and terminates the session.

flight $_{\text {two }}$ information is generated by the Edge Router and communicated with a sensor which claims authentication for session keys. Edge Router ID and nonce value is encrypted with flight $t_{t w o}$ key SK2 and $M A C_{t w o}$ is calculated by using a hash function on the resultant encrypted values. Along with the encrypted values and $M A C_{t w o}$ values, "ERConf" message is sent to the sensor as a second flight information. After receiving flight $t_{\text {two }}$ information, sensor checks the $M A C_{t w o}$ value by calculating the same with the procedure followed by the Edge Router for $M A C_{t w o}$ calculation. If the $M A C_{t w o}$ is not replayed by any adversaries, then the sensor generates flight $_{\text {two }}$ key SK2 and decrypt the received flight two $_{\text {in- }}$ formation from the Edge Router. After the above steps are performed, the sensor stores the ID of the Edge Router.

With the retrieved values from flight $t_{\text {two }}$ packet, the sensor generates flight $t_{\text {three }}$ key SK3 by applying XOR functions between Edge Router ID and flight two $_{\text {key SK2. flight }}$ three information are generated by encrypting the old and new nonce value of sensor and flight $t_{\text {two }}$ key, with flight $_{\text {three }}$ key. Then $M A C_{\text {three }}$ is calculated by applying a hash function on the encrypted values. Now flight three information packet is ready to send to Edge Router along with the "Sensor Challenge" message. When the Edge Router receives flight three $_{\text {infor- }}$ mation from sensor, Edge Router checks whether it has sent flight $_{\text {two }}$ information to the intended sensor.

Upon getting positive results of the checking operation, Edge Router starts to process the received flight three $_{\text {infor- }}$ mation from sensors. Initially Edge Router checks $M A C_{\text {three }}$ value by calculating it and compare with the received $M A C_{\text {three }}$ value. If the Edge Router found, the packet is not replayed, then proceed to calculate flight $t_{\text {three }}$ key SK3 otherwise, terminates the session by sending "You have replayed the message".

flight $_{\text {three }}$ information are decrypted using flight $t_{\text {three }}$ key SK3 and Edge Router gets the information like old, the new nonce value of sensor and flight $t_{\text {two }}$ key SK2 calculated by the sensor. Edge Router does the second level authentication by comparing the nonce value of sensor what it has received from flight $_{t w o}$ and comparing flight two $_{\text {twey SK2 }}$ value with the existing information. If the value matches, then it starts to process the further required session key generation. Thus the authentication phase of the sensor is completed by the Edge Router.

3) Key Distribution phase: $6 L_{E R} \longrightarrow 6 \mathrm{~L}_{S}$ :

Finished, ( $\left.\left(K_{\text {Session }}\right) \mathrm{SK} 4\right), \mathrm{MAC} 4$

$\mathrm{MAC} 4=\mathrm{H} \quad\left[\left(\left(K_{\text {Session }}\right) \mathrm{SK} 4\right), \mathrm{SK} 4\right]$

flight $_{\text {four }}$ key SK4 is the composition of old and new nonce values of the sensor and then the flight $t_{\text {three }}$ key SK3. The session key ( $\left.K_{\text {Session }}\right)$ is composed of flight $t_{\text {one }} \mathrm{SK} 1$, flight $_{\text {two }}$ SK2, flight three $_{\text {SK3, flight }}$ four SK4 and nonce of Edge Router at the time of session key generation. The session key is generated by the Edge Router by applying the XOR function on the above said values. The session 


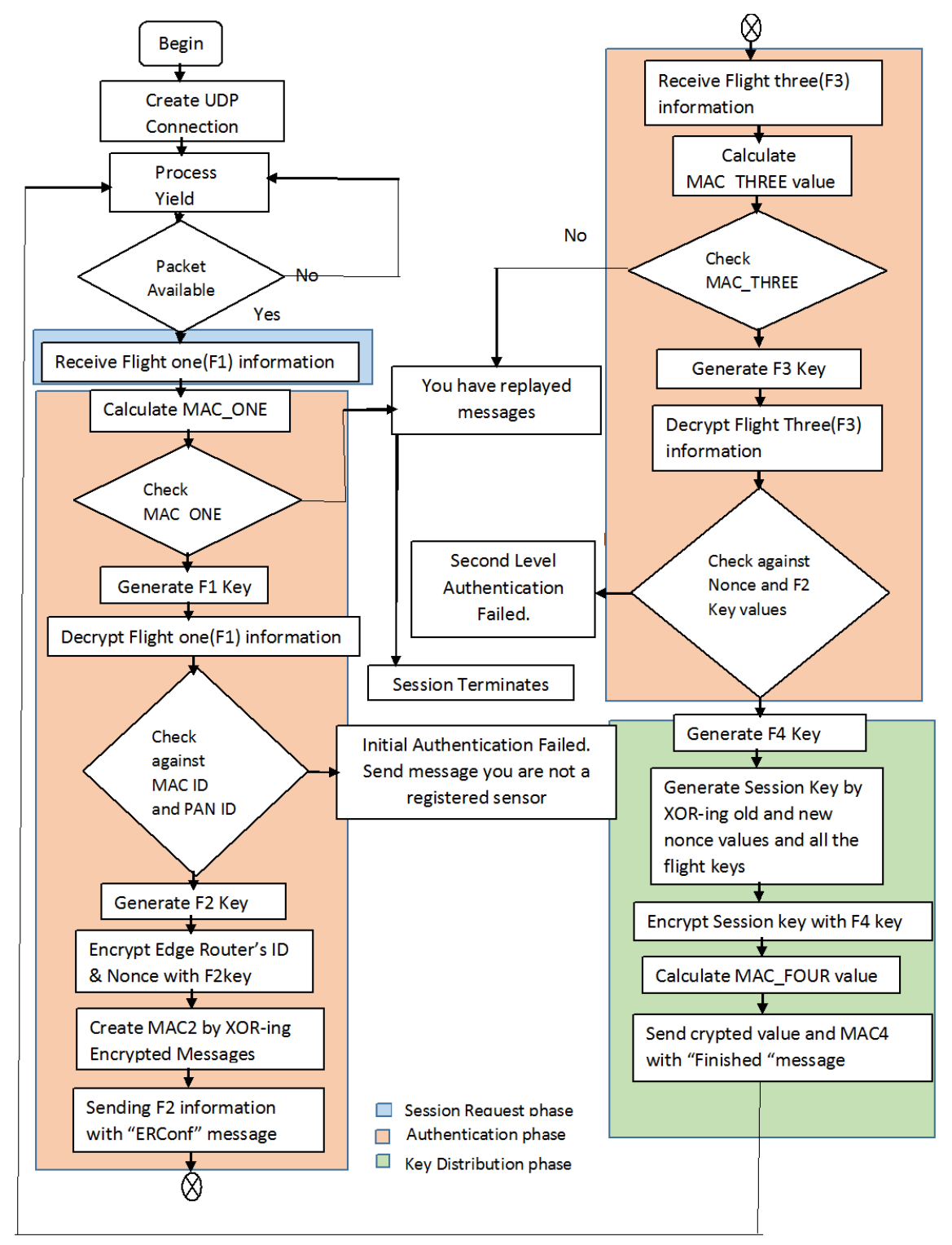

Fig. 4: Flow chart of Edge Router Process

key is encrypted with flight $_{\text {four }}$ key SK4 and is generated by the Edge Router. $M A C_{\text {four }}$ value is calculated using XOR functions on encrypted values and flight four $_{\text {key }}$ SK4. After the cryptographic functions and $M A C_{\text {four }}$ calculation, flight $_{\text {four }}$ information is sent to the intended sensor with the "Finished" message.

flight $_{\text {four }}$ information are received by the sensor and sensor generates flight $_{\text {four }}$ key SK4 using the same method followed by the Edge Router. A sensor checks whether the message is replayed or not by checking the $M A C_{\text {four }}$ value with the calculated $M A C_{\text {four }}$. If the flight $_{\text {four }}$ message packet, through the checking operation of MAC values, then the sensor decrypts the flight $_{\text {four }}$ message and get the session key $\left(K_{\text {Session }}\right)$. Thus the key distribution process is completely done by the Edge Router to the sensor by means of communicating four flight messages. This session key is used as a key to encrypt the further communication.

\section{FORMAL VERIFICATION OF AlgORITHM}

The Scyther formal verification tool is used to verify the authentication properties of our proposed algorithm. Figure 5 shows the result of the LAUP algorithm using a formal authentication verification tool called Scyther.

Definitions of Aliveness, Secrecy, the NonInjective - Agreement, and Non-Injective-Synchronization are defined in [20], [21]. Figure 5 result shows LAUP algorithm satisfies all the specified authentication properties.

Secrecy expresses that certain information is not revealed to an intruder, even though we are communicating this data over an untrusted network. By maintaining the secrecy of Edge Router ID, we can perform a second level authentication of 6LoWPAN devices with Edge Router also the intruder can not 
get any information of Edge Router ID.

Non-Injective Synchronization property of 6LoWPAN sen-

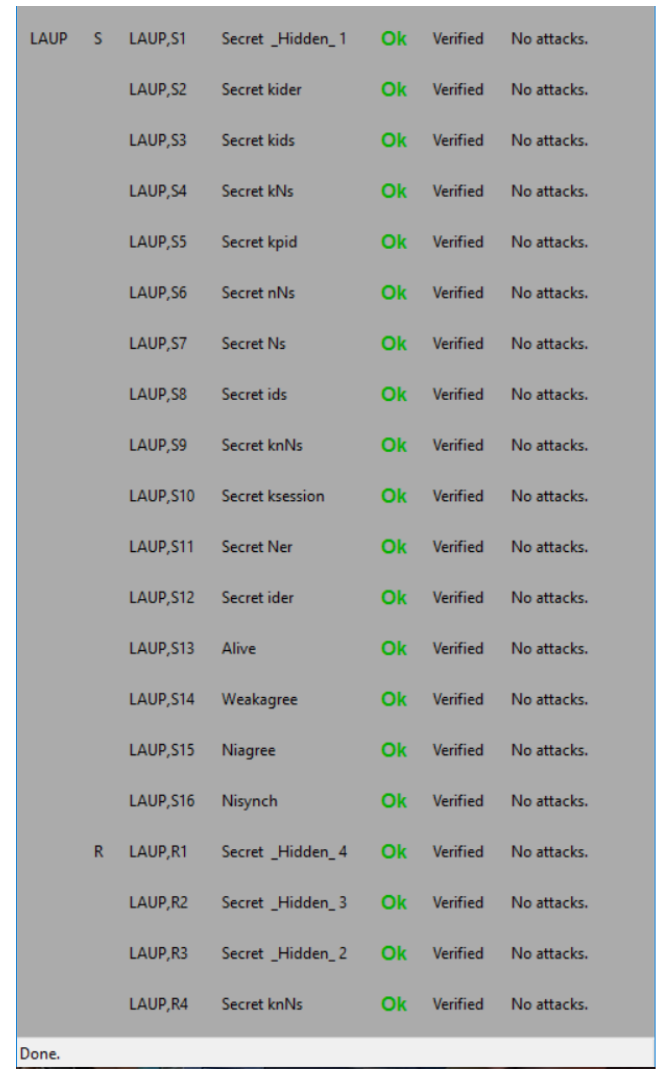

Fig. 5: Automatic Authentication verification by Scyther

sor, ensures that it communicates with the intended party 6LoWPAN Edge Router and the contents of receiving/sending messages are equal. Also, it guarantees the expected order of send and receives actions.

\section{SECURITy ANALYSIS}

The session key establishment and authentication method followed by LAUP algorithm are well suited for LoWPAN wireless network sensors. Because,the LAUP algorithm uses lightweight symmetric cryptographic methods to establish a session key and authentication process. Since the MAC address of the 6LoWPAN sensor device and Edge Router are in the encrypted form during the process of LAUP, it will not be disclosed to an eavesdropper.

The proposed LAUP algorithm gives reliable protection against the well known LoWPAN security attacks.

REPLAY ATTACK: LAUP protects the transmission of messages from replay attack in all the four flights by adding MAC values, thereby the integrity of the message is maintained throughout the algorithm. So insertion, deletion or modification of messages could not be performed by the attacker. All the four flight information analyzed step by step for what would happen if the attacker captures the flight information. The first flight message packet could not be reproduced by the attacker because the packet contains information such as sensor' $s$ unique MAC ID and the timer value of sensor at the time of first flight message generation and most importantly they are appended with $M A C_{\text {one }}$ value. The second flight message contains the nonce value of Edge Router encrypted with the unique flight $_{t w o}$ key SK2. Also, we proved the secrecy of Edge Router ID with Scyther tool, so that adversaries cannot get this information and reproduce it.

This strongly encrypted value cannot be deciphered by the attacker since he does not know the nonce value of Edge Router. The third flight message contains nonce values used in the first flight and the nonce value of the third flight, encrypted with unique flight $t_{\text {three }}$ key SK3. These ciphertexts are cryptographically strong enough for the lightweight communication and cannot be replayed so that the integrity of the message maintained. The fourth flight message has $M A C_{\text {four }}$ value and ciphered form of the session key. An attacker can get the session key only if he knows unique flight $t_{\text {four }}$ key SK4. On the whole, nonce values and MAC values prevent the attacker from replaying the message and maintaining integrity.

MAN IN THE MIDDLE ATTACK: LAUP protects the communication of messages against Man in the Middle attack. The man in the middle attacker possibly alters the communication between the two parties who believe that they are directly communicating with each other. But LAUP messages, in all four flights, are encrypted with the secure AES-128-ECB algorithm and unique flight keys.The flight messages are constructed with nonce values. Also, Non-Injective synchronization property is maintained.

IMPERSONATION ATTACK: Here an adversary can pretend like one of the legitimate sensors in the LoWPAN network. LAUP assumes all the sensors' identity are registered with the Edge Router. Sensor hello request from an impersonation adversary rejected by checking its identity.

\section{Vi. Evaluation of Proposed AUP Algorithm}

Our proposed LAUP algorithm for authentication and key distribution algorithm simulated in Contiki OS COOJA simulator environment. Our simulated environmental architecture is shown in Figure 6. We have taken Wismote as a sensor and the Edge Router as well. The scalability of our proposed LAUP algorithm is checked by adding 65 nodes to the network with the Edge Router. LAUP simulated like an rpl udp client server application whereas an Edge Router acts as a server.

Wismote voltage value and various current values such as CPU current value, low power mode current value, transmission, and reception current values are taken from the Wismote datasheet [22]. Power consumption is calculated using the formula found in [23]. The sensor who wants to communicate with the Edge Router is consuming $0.0456 \mathrm{mw}$ of CPU power, $0.0048 \mathrm{mw}$ of low power mode (lpm) power, $0.1567 \mathrm{mw}$ of transmission power, $0.3300 \mathrm{mw}$ of reception power and 0.5371 mw of total power. We simulated our algorithm with ten sensors. The graph in Figure 7 explains the comparison of computational overhead of LAUP with EAKES6Lo and SAKES [14] overhead values given in [9]. Although we compared the authentication algorithms (EAKES6Lo, SAKES) which 
TABLE III: Memory usage of sensor and Edge Router

\begin{tabular}{llllll}
\hline text & data & bss & dec & hex & filename \\
\hline 45619 & 350 & 13236 & 59205 & e745 & udp-clientv1.wismote \\
49253 & 402 & 13782 & 63437 & f7cd & border-routerv2.wismote \\
\hline
\end{tabular}

simulated in different environments, the LAUP authentication algorithm provides 15 times less computational overhead than EAKES6lo and 18 times less computational overhead than SAKES authentication algorithm for LoWPAN devices. Figure 9 shows the power consumption value increases as the number of nodes increases also this graph tells us power consumption while receiving messages is high compared to the $1 \mathrm{pm}$, and transmission energy consumption in LoWPAN devices.

The difference in power consumption of conventional sensor (without any authentication) and LAUP sensor (with the proposed authentication algorithm) is explained in Figure 8, and LAUP consumes $0.13079624 \mathrm{mw}$ more power while transmitting messages than the regular sensor communication without authentication. Each flight of LAUP is communicated as a payload of the transport layer. Flight 1 (SensorHello) and Flight 2 (ERConf) consume 64 bytes each. Flight 3 (SensorChallenge) consumes 80 bytes. Flight 4 (Finished) consumes 48 bytes. Graphs in Figure 10 reveals the total processing time of the LAUP algorithm for different sensors over time. From this graph, taking the average of the total processing time of 10 sensors, we proved that our proposed LAUP algorithm takes less time to execute the full authentication algorithm. Up to 65 6LoWPAN devices can be connected without resourceexhaustion to the Edge Router in a specific position. Coding of LAUP will be sent to the reader upon request.

Memory usage of LAUP algorithm is calculated on the sensor, and the Edge Router based on the information found in [24]. Table III summarizes the memory usage of the sensor and the Edge Router. Data segment refers read-write data, and bss segment indicates zero initialized data. The sum of text, data and bss values mentioned in dec section. Flash consumption of LAUP algorithm is 45969 bytes in sensor and 49655 bytes in the Edge Router. RAM use of LAUP algorithm is 13586 bytes in sensor and 14184 bytes in the Edge Router. The total processing time of our proposed LAUP algorithms takes $421.3 \mathrm{msec}$ which is comparatively lower than the processing time of existing algorithms such as EAKES6Lo and SAKES are given in [9].

\section{CONCLUSION}

With the knowledge of existing algorithms and their limitations in the field of authentication and key distribution, we proposed our LAUP algorithm to overcome these limitations. Our algorithm formally verified by the formal verification tool called "Scyther", and we proved that authentication properties such as Aliveness, NonInjective Agreement, Secrecy of keys and Non-Injective Synchronization are maintained. Moreover, LAUP algorithm works with UDP protocol and possible threats such as Replay attack, Man in the Middle attack and

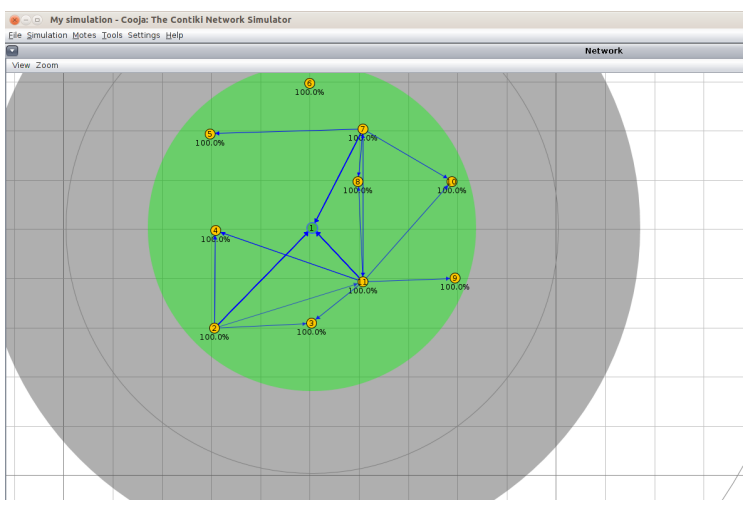

Fig. 6: Simulation Scenario implementing LAUP

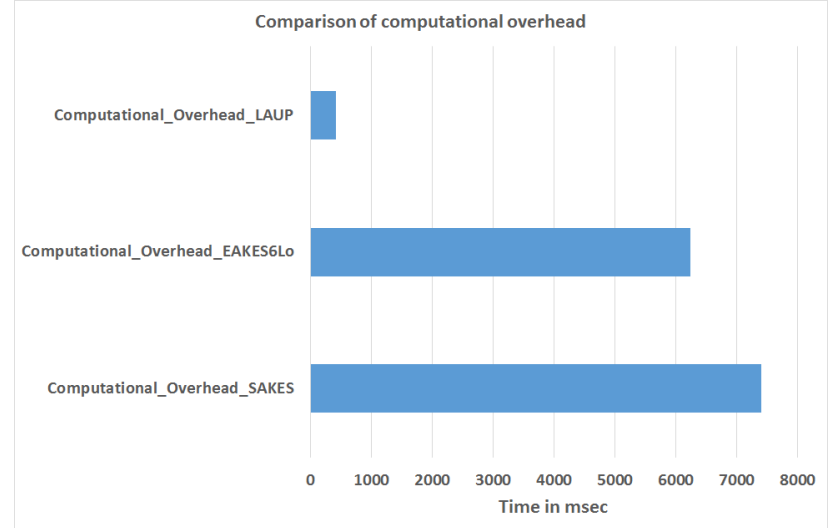

Fig. 7: Comparison of computational Overhead

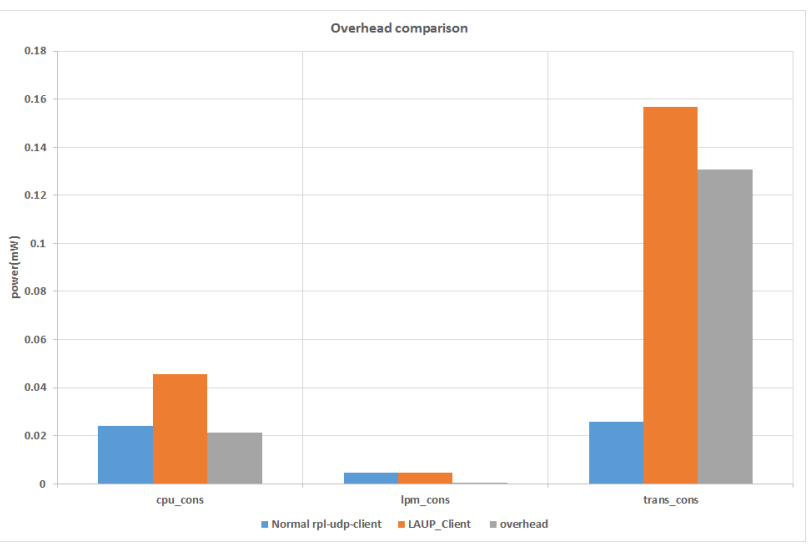

Fig. 8: Powerconsumption overhead

impersonation attack are analyzed theoretically in section $\mathrm{v}$. In addition to the formal verification proof, we presented simulation results using the Contiki OS COOJA simulator with 


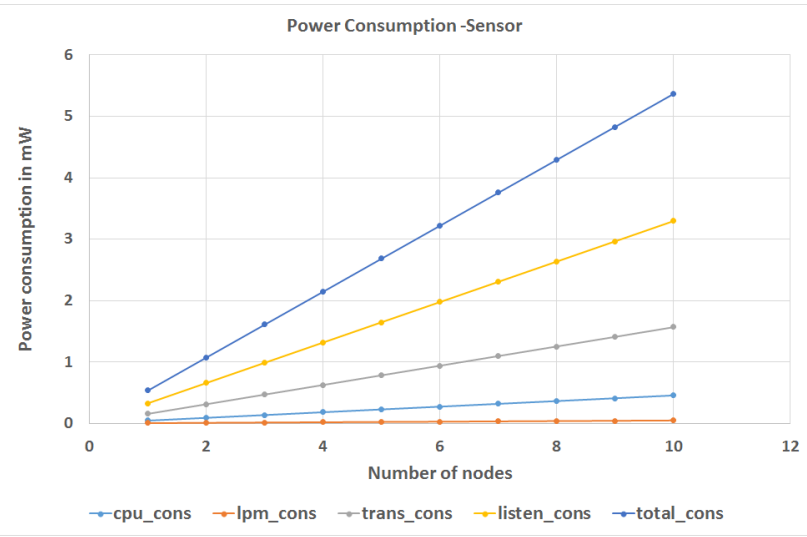

Fig. 9: Power Consumption based on number of Nodes

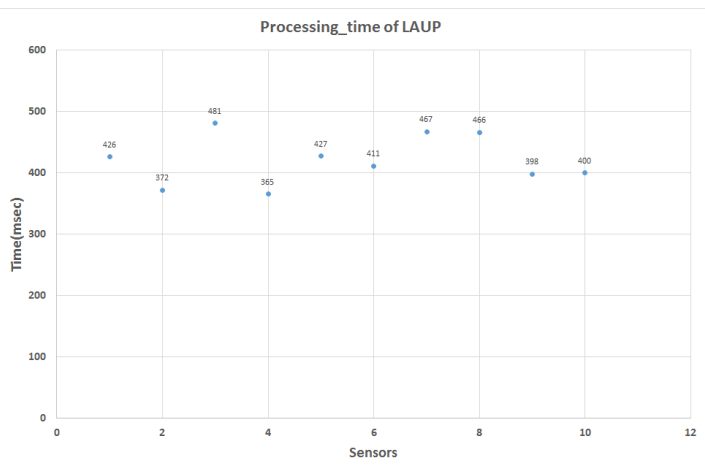

Fig. 10: Total processing time using LAUP

ten 6LoWPAN sensors as clients and one Edge Router. The simulation results broadly supported theoretical predictions. Evaluation of the proposed algorithm carried out based on the simulation time. From our evaluation results, we can say that our algorithm is highly secured since LAUP generates the respective keys for each flight using the nonce value of sensors and Edge Router. Additionally, this LAUP algorithm is flexible to update the keys after each session. In future, we will deploy our LAUP using a testbed and compare the result with the simulation results. Also, LAUP will be tested against Sybil attacks using Cooja simulator and the hardware. From the verification tool result and the evaluation result of the simulation, we proved that the LAUP algorithm for authentication and key distribution is highly secured, scalable for LoWPAN networks and flexible enough to update the keys.

\section{REFERENCES}

[1] Z. Shelby and C. Bormann, 6LoWPAN: The wireless embedded Internet John Wiley \& Sons, 2011, vol. 43.

[2] R. Mahmoud, T. Yousuf, F. Aloul, and I. Zualkernan, "Internet of things (iot) security: Current status, challenges and prospective measures," in 10th International Conference for Internet Technology and Secured Transactions (ICITST). IEEE, 2015, Conference Proceedings, pp. 336341.

[3] A. Al-Fuqaha, M. Guizani, M. Mohammadi, M. Aledhari, and M. Ayyash, "Internet of things: A survey on enabling technologies, protocols, and applications," Communications Surveys \& Tutorials, IEEE, vol. 17, no. 4, pp. 2347-2376, 2015.
[4] Z. Sheng, S. Yang, Y. Yu, A. Vasilakos, J. Mccann, and K. Leung, "A survey on the ietf protocol suite for the internet of things: Standards, challenges, and opportunities," IEEE Wireless Communications, vol. 20, no. 6, pp. 91-98, 2013.

[5] L. Gheorghe, R. Rughinis, R. Deaconescu, and N. Tapus, "Authentication and anti-replay security protocol for wireless sensor networks," in Systems and Networks Communications (ICSNC), 2010 Fifth International Conference on. IEEE, 2010, pp. 7-13.

[6] A. J. Jara, L. Marin, A. F. Skarmeta, D. Singh, G. Bakul, and D. Kim, "Mobility modeling and security validation of a mobility management scheme based on ecc for ip-based wireless sensor networks (6lowpan)," in Innovative Mobile and Internet Services in Ubiquitous Computing (IMIS), Fifth International Conference on. IEEE, 2011, Conference Proceedings, pp. 491-496.

[7] N. J. Al Fardan and K. G. Paterson, "Lucky thirteen: Breaking the tls and dtls record protocols," in Security and Privacy (SP), IEEE Symposium on. IEEE, 2013, Conference Proceedings, pp. 526-540.

[8] S. Raza, T. Voigt, and V. Jutvik, "Lightweight ikev2: a key management solution for both the compressed ipsec and the ieee 802.15. 4 security," in Proceedings of the IETF workshop on smart object security, vol. 23. Citeseer, 2012, Conference Proceedings.

[9] Y. Qiu and M. Ma, "A mutual authentication and key establishment scheme for $\mathrm{m} 2 \mathrm{~m}$ communication in 6lowpan networks," IEEE Transactions on Industrial Informatics, vol. 12, no. 6, pp. 2074-2085, 2016.

[10] S. Raza, D. Trabalza, and T. Voigt, "6lowpan compressed dtls for coap," in Distributed Computing in Sensor Systems (DCOSS), 2012 IEEE 8th International Conference on. IEEE, 2012, pp. 287-289.

[11] K.-F. Krentz, H. Rafiee, and C. Meinel, "6lowpan security: adding compromise resilience to the 802.15 .4 security sublayer," in Proceedings of the International Workshop on Adaptive Security. ACM, 2013, p. 1.

[12] A. Perrig, R. Szewczyk, J. D. Tygar, V. Wen, and D. E. Culler, "Spins: Security protocols for sensor networks," Wireless networks, vol. 8, no. 5, pp. 521-534, 2002.

[13] K.-F. Krentz and C. Meinel, "Handling reboots and mobility in 802.15. 4 security," in Proceedings of the 31st Annual Computer Security Applications Conference. ACM, 2015, pp. 121-130.

[14] H. R. Hussen, G. A. Tizazu, M. Ting, T. Lee, Y. Choi, and K.-H. Kim, "Sakes: Secure authentication and key establishment scheme for $\mathrm{m} 2 \mathrm{~m}$ communication in the ip-based wireless sensor network (610wpan)," in Fifth International Conference on Ubiquitous and Future Networks (ICUFN). IEEE, 2013, pp. 246-251.

[15] M. Li, S. Yu, J. D. Guttman, W. Lou, and K. Ren, "Secure ad hoc trust initialization and key management in wireless body area networks," $A C M$ Transactions on sensor Networks (TOSN), vol. 9, no. 2, p. 18, 2013.

[16] R. D. Pietro, D. Ma, C. Soriente, and G. Tsudik, "Self-healing in unattended wireless sensor networks," ACM Transactions on Sensor Networks (TOSN), vol. 9, no. 1, p. 7, 2012.

[17] S. N. Premnath and Z. J. Haas, "Security and privacy in the internet-ofthings under time-and-budget-limited adversary model," Wireless Communications Letters, IEEE, vol. 4, no. 3, pp. 277-280, 2015.

[18] C. Hennebert and J. Dos Santos, "Security protocols and privacy issues into 6lowpan stack: A synthesis," Internet of Things Journal, IEEE, vol. 1 , no. 5, pp. 384-398, 2014.

[19] A. Mukherjee, "Physical-layer security in the internet of things: Sensing and communication confidentiality under resource constraints," Proceedings of the IEEE, vol. 103, no. 10, pp. 1747-1761, 2015.

[20] C. J. F. Cremers, Scyther: Semantics and verification of security protocols. Eindhoven University of Technology, 2006.

[21] G. Lowe, "A hierarchy of authentication specifications," in Computer security foundations workshop, 1997. Proceedings., 10th. IEEE, 1997, pp. 31-43.

[22] T. Instruments, "Cc2520 datasheet, 2007," 2014

[23] SonHan, "Thigschat Internet of things," http://thingschat.blogspot.com. au/2015/04/contiki-os-using-powertrace-and.html/, 2016, [Online; accessed 12-November-2016].

[24] A. Velinov and A. Mileva, "Running and testing applications for contiki os using cooja simulator," 2016. 